Asientos corológicos 25. Cavanillesia altera 2: 127-128. Madrid.

GAMARRA, R. y Ó. MONTOUTO -1994- Mapa 671. In: F. J. Fdez Casas et al. (eds.) Asientos para un atlas corológico de la Flora Occidental 22. Fontqueria 40: 211-214.

MOLERO, J. -1990- Microcnemum ung. Sternb. In: Castroviejo et al. (eds.) Flora Iberica Vol. II. Real Jardín Botánico. CSIC. Madrid. MOLINA ABRIL, J.A., C. PERTÍÑEZ y M.T. DE LA CRUZ -2001- Datos sobre la relación suelo-vegetación en los saladares de Cordovilla (Albacete, España). Sabuco 1 (1): 217-232. Albacete.

RIVAS-MARTÍNEZ, S.et al. -2002 - Vascular plant communities of Spain and Portugal. Addenda to the Syntaxonomical checklist of
2001. Itinera Geobotanica 15: 5-922. Madrid.

VALDÉS-FRANZI, A., F. ALCARAZ y D. RIVERA -2001 - Catálogo de las plantas vasculares de la provincia de Albacete. Instituto de Estudios Albacetenses, Serie I, 127. Albacete.

Aceptado para su publicación en octubre de 2004

Dirección de los autores. P. CANTÓ: Departamento de Biología Vegetal II, Facultad de Farmacia, Universidad Complutense. E-28040 Madrid, Spain; F. ALCARAZ: Departamento de Biología Vegetal, Universidad de Murcia, E30100 Murcia, Spain.

\title{
143. DATOS TAXONÓMICOS Y SINTAXONÓMICOS DE LAS ESPECIES DEL GÉNERO PUCCINELLIA PARL. (POACEAE) EN EL SURESTE DE LA PENÍNSULA IBÉRICA
}

\author{
$\mathrm{M}^{\mathrm{a}}$ Ángeles ALONSO y Antonio DE LA TORRE
}

Taxonomic and syntaxonomic study of the species of genus Puccinellia Parl. in the southeastern of Iberian Peninsula.

Palabras clave. Puccinellia, fitosociología, nomenclatura, Península Ibérica.

Key words. Puccinellia, phytosociology, nomenclature, Iberian Peninsula.

El género Puccinellia Parl. (Poaceae) ha sido desde siempre un género muy controvertido taxonómicamente. No fue hasta que Julià (1992) publicara una monografía de este género para la Península Ibérica, donde se presentaban claves de determinación y mapas de distribución de las especies peninsulares, cuando se han podido

Este trabajo ha sido financiado parcialmente gracias al proyecto de investigación GV99-39-1-09 de la Generalitat Valenciana. 
determinar con fiabilidad algunos táxones de este complicado género. De este modo, la presencia de algunos táxones citados en la bibliografía se ha puesto en duda. Tal es el caso de Puccinellia distans (L.) Parl. mencionado por Rigual (1972), Alcaraz (1984), Alonso (1996), Vicedo \& De la Torre (1997) y Puccinellia festuciformis (Host) Parl. citado en Alonso (1996), o Mateo \& Crespo (2001). Esta problemática ha obligado a un estudio de los taxones en profundidad para abordar el estudio sintaxonómico, ya que, la controversia producida por la errónea determinación de las especies ha provocado diferentes errores en la sintaxonomía que se han ido solventando en base a nuevas propuestas taxonómicas (Alonso \& De la Torre, 2003).

Para la correcta determinación de los pliegos se ha seguido la monografía de Juliá (1992). Para hacer un estudio corológico de las diferentes especies del género Puccinellia, se han revisado los pliegos de este género de los herbarios de la Universidad de Alicante (ABH), Universidad de Murcia (MUB), Universidad de ValenciaJardín Botánico (VAL) y algunos pliegos del herbario del Jardín Botánico de Madrid (MA), correspondientes a las provincias de Alicante, Albacete y Murcia (ver Anexo I).

El estudio de las comunidades vegetales ha sido realizado con la metodología fitosociológica de la escuela de ZürichMontpellier (Géhu \& Rivas Martínez ,1982).

El tratamiento sintaxonómico se ha realizado al amparo del Código de Nomenclatura Fitosociológica (Weber et al., 2000).

\section{Aspectos taxonómicos}

Después de un estudio de pliegos de herbario y de material recolectado se puede concluir que en el sureste de la Península se encuentran dos especies de Puccinellia: Puccinellia fasciculata (Torr.) E. P. Bicknell y Puccinellia caespitosa G. Montserrat \& J. M. Montserrat. La primera es la más extendida y se instala sobre suelos salinos siempre húmedos. La segunda, ocupa una ecología algo diferente, ya que se instala en suelos salinos que han sufrido desecación, es más rara que la anterior y se localiza sobre todo en las lagunas salinas manchegas. Las poblaciones más grandes del sureste se encuentran en la laguna de Pétrola (Albacete). Se cita en este trabajo una nueva localidad de Puccinellia caespitosa G. Montserrat \& J. M. Montserrat de la zona de Villena (Alicante), que se encontró revisando la colección de Rigual del herbario $\mathrm{ABH} y$ que se ha vuelto a recolectar en la misma localidad.

\section{Aspectos sintaxonómicos}

Una vez solventado los errores nomenclaturales producidos por la errónea identificación de algunas especies de este género (Alonso \& De la Torre, 2003). Se propone una nueva asociación que englobaría los herbazales de Puccinellia fasciculata que se instalan sobre suelos siempre húmedos y que pueden ser monoespecíficas o a menudo acompañados de especies halófilas como Spergularia media o Aeluropus littoralis.

\section{Spergulario mediae-Puccinellietum} fasciculatae Alonso \& De la Torre ass. nova.

Tabla1, inv. 1-9, Holotypus: inv. 6

Praderas halófilas pobres en especies, dominadas principalmente por Puccinellia fasciculata, en algunas ocasiones acompañada por otras gramíneas como Aeluropus littoralis y muy a menudo por la vivaz Spergularia media. La comunidad tiene un carácter pionero, coloniza taludes húmedos 
Tabla 1

\begin{tabular}{|c|c|c|c|c|c|c|c|c|c|}
\hline \multirow[b]{2}{*}{$\mathrm{N}^{\circ}$ Orden } & \multicolumn{9}{|c|}{$\begin{array}{l}\text { Spergulario mediae-Puccinellietum fasciculatae } \\
\text { Alonso \& De la Torre ass. nova } \\
\text { a maritimae, Juncetalia maritimae, Puccinellion fasciculatae) }\end{array}$} \\
\hline & 1 & 2 & 3 & 4 & 5 & 6 & 7 & 8 & 9 \\
\hline Altitud (m) & 960 & 660 & 600 & 540 & 500 & 1 & 290 & 260 & 100 \\
\hline Superficie $(\mathrm{m} 2)$ & 30 & 30 & 20 & 12 & 10 & 10 & 3 & 4 & 10 \\
\hline \multicolumn{10}{|l|}{ Combinación h abitual } \\
\hline Puccinellia fasciculata & 5 & 4 & 2 & 5 & 4 & 4 & 5 & 1 & 4 \\
\hline Aeluropus littoralis & 1 & - & 1 & - & - & - & - & 4 & - \\
\hline Spergularia media & - & - & - & 1 & - & 2 & - & - & 2 \\
\hline Plantago serpentina & - & - & + & - & - & - & - & - & - \\
\hline \multicolumn{10}{|c|}{ Caract. clase, orden, alianza } \\
\hline Sonchus maritimus & - & - & - & - & + & - & - & - & - \\
\hline Cotula coronopifolia & - & - & - & - & - & + & - & - & - \\
\hline $\begin{array}{l}\text { Juncus subulatus } \\
\text { Compañeras }\end{array}$ & - & - & - & - & - & - & - & + & - \\
\hline Phragmites australis & - & - & - & + & - & + & - & - & + \\
\hline Suaeda vera & - & - & - & 1 & - & - & - & - & - \\
\hline Aster squamatus & - & 1 & - & - & - & - & - & - & - \\
\hline Scirpus maritimus & + & - & - & - & - & - & - & - & - \\
\hline Tamarix canariensis & - & + & - & - & - & - & - & - & - \\
\hline Limonium delicatulum & - & - & - & - & - & - & + & - & - \\
\hline
\end{tabular}

Localidades: 1. Laguna de Pétrola, XJ2000 (Ab). 2.- Salinas de Fuentealbilla, XJ2547 (Ab). 3.Cordovilla, XH1671 (Ab). 4.- Base del Cabezo de la Sal, Pinoso, XH7148 (A). 5.- Acequia del Rey. Villena, XH8176 (A).6.- Barranco de las Ovejas, Alicante, YH1745 (A). 7.- Río Vinalopó, Novelda, XH9723 (A). 8.- Rambla de la Parra, XH6733 (Mu). 9. Rambla del Ajauque, XH6642 (Mu). 
y depresiones con un carácter nitro-halófilo y con un periodo de inundación variable. Es una asociación, termo y mesomediterránea, con amplia distribución en el sureste.

\section{Esquema sintaxonómico}

Se presenta a continuación un esquema sintaxonómico con todas las asociaciones peninsulares en las que aparecen las especies del género Puccinellia.

Cl. Juncetea maritimi Br.-Bl. in Br.-Bl., Roussine \& Nègre 1952

Ord. Juncetalia maritimi Br.-B1. ex Horvatic 1934

All. Puccinellion lagascanae RivasMartínez in Rivas-Martínez \& Costa 1976 corr. Alonso \& De la Torre 2003

Spergulario mediae-Puccinellietum fasciculatae Alonso \& De la Torre ass. nova

Puccinellietum lagascanae

Rivas Goday 1955

corr. Alonso \& De la Torre 2003

Puccinellio fasciculatae-Artemisietum gargantae Cirujano 1981 corr. Alonso \& De la Torre 2003

Artemisio gargantae-Puccinellietum pungentis Barrera et. Cirujano 1986 corr. Rivas Martínez et al. 2001

Bupleuro tenuissimi-Juncetum gerardii Rivas Martínez in Rivas Martínez \& Costa 1976

Plantagini maritimae-Camphorosmetum monspeliacae Ladero et al. 1984

\section{Anexo 1: material estudiado}

Puccinellia fasciculata (Torrey) Bickwell

ALICANTE: Alicante, el barranco de las Ovejas, desembocadura, 1 m, YH1746, 5-5-1999, M.A. Alonso \& J.J. Montoya (ABH 41905).

Crevillente, carretera Crevillente-Catral, 15 m, XH9130, M.A. Alonso, A. De la Torre \& M. Vicedo (ABH 45453).
Elche, Pantano del Vinalopó, suelo salino encharcado, 7-5-193, F. Alcaraz (MUB10056)

Altea, playa del Albir, A. Rigual, 22-5-1958 (VAL 138008)..

Jijona, barranco del Almadén, A. Rigual, 195-1959 (VAL 138006).

Villena, camino del Cabezo Redondo, A. Rigual, 16-4-1960 (VAL 138007).

Novelda, Cauce del Vinalopó, A. Rigual, 115-1961 (VAL 38510). .

Crevillente, E1 Hondo, XH92; 18-1-1981, F. Alcaraz (MUB4105)

Xabia, Riu Gorgos, 20 m, BC5192, 10-1992, A. Barber (ABH 4593).

Alicante, barranco de las Ovejas, $50 \mathrm{~m}$, YH1548, 26-2-1994, J.C. Cristobal (ABH 12233).

Tibi, río Verde, próximo Pinar Terol, $490 \mathrm{~m}$, YH0996, 4-5-1994, JC. Cristobal (ABH 12300).

Crevillente, barranco de la Garganta, $200 \mathrm{~m}$, XH9238, 20-5-1994, M. Vicedo, De la Torre, M.A. Alonso (ABH 121332).

Villena, acequia del Rey, 500 m, XH8078, 24-11-1994, M. A. Alonso, De la Torre \& M. Vicedo (ABH 12677).

Crevillente, San Pascual, 200 m, XH9034, 11-1-1995, M. Vicedo, A. De la Torre, M. A. Alonso (ABH 12693).

Sax, cauce del río Vinalopó, $490 \mathrm{~m}$, XH9068, 11-4-1995, M.A. Alonso, A. De la Torre \& M. Vicedo (ABH 13971).

Sax, cauce del río Vinalopó, $490 \mathrm{~m}$, XH9068, 11-4-1995, M.A. Alonso, A. De la Torre \& M. Vicedo (ABH 13973).

Crevillente, Embalse, 150 m, XH9337, 36-1995, M. Vicedo \& A. De la Torre, .

Elda, Pantano de Elda, 540 m, XH9163, 2910-1995, A. Juan (ABH 15371).

Pinoso, Cerro de la Sal, 590 m, XH7148, 26-1996, M.A. Alonso \& J.J. Montoya (ABH 30550).

Pinoso, Cerro de la Sal, 650m, XH7348, 26-1996, A. Navarro \& J. Escandell (ABH 31704 ).

Alicante, barranco de las ovejas, $70 \mathrm{~m}$, YH1550, 1-10-1996, E. Camuñas \& M.B. Crespo (ABH 30721).

Alicante, barranco de las ovejas, $1 \mathrm{~m}$, YH1745, 6-10-1996, E. Camuñas \& M.B. Crespo (ABH32385).

Alicante, La Albufera, 10 m, YH2349, 19- 
1-1997, E, Camuñas \& M.B. Crespo (ABH 35219).

Elche, rambla de la Goteta, $80 \mathrm{~m}$, XH9839, 18-3-1997, M.A. Alonso, A. Dela Torre, M. Vicedo \& Ortega (ABH 33655).

Novelda, río Vinalopó, 290 m, XH9723, 135-1997, M.A. Alonso \& J.J. Montoya (ABH 42521).

Crevillente, El Fondó, El codo, $10 \mathrm{~m}$, XH9429, 9-12-1997, L. Serra \& A. Olivares (ABH 40587).

Alicante, Villafranqueza, $90 \mathrm{~m}$, YH1952, 22-9-1998, E. Camuñas \& M.B. Crespo (ABH 40993).

Rojales, 0 m, XH9919, 6-10-2000, A. Juan, M. Fabregat, M. Ocampo \& Ruiz de León (ABH 44153).

Alicante, La Albufereta, playa bahía los Pinos, 1 m, YH2449, 12-1-1997, E. Camuñas \& M. B. Crespo (ABH 32170).

Alicante, barranco de las ovejas, S. Gabriel, 10 m, YH14, 20-3-1996, De la Torre, M. Vicedo \& M.A. Alonso (ABH16918).

MURCIA: Cieza, Rambla del Moro, XH4232, 26-4-1981, F. Alcaraz (MUB5725)

Jumilla, Charco del zorro, margen de corriente de agua algo salino, $480 \mathrm{~m}, \mathrm{XH} 4560$, 1-5-1982, F. Alcaraz (MUB6948)

Abanilla, Río Chicamo, suelos salinos, 160 m, XH7028, 8-5-1982, F. Alcaraz (MUB8071)

ALBACETE: Pétrola, 960 m, XJ2000, 2-51999, M.A. Alonso, M.D. Vargas \& J. Alonso (ABH41909)

Pétrola, 9-6-181, D. Rivera (MUB 39810)

La Higuera, Laguna del Saladar, 27-5-1980, D. Rivera (MUB39809)

Puccinellia caespitosa G. Monserrat \& J. M: Monserrat

ALICANTE: Villena, Prados de Galeno, sobre arcillas algo salobres, A. Rigual ut Festuca scariosa (MA 311017)

Villena, Prados de Galeno, 500 m, XH 8774, 31-10-2003, sobre arcillas salobre, M. A. Alonso, J. Rapo \& J. Montoya (ABH 47147)

ALBACETE: Pétrola, 960 m, XJ2000, 2-51999, M.A. Alonso, M.D. Vargas \& J. Alonso (ABH 41909)

\section{BIBLIOGRAFÍA}

ALONSO, M. A. y A. DE LA TORRE -2003Precisiones nomenclaturales sobre los gramales halófilos (All. Puccinellion Rivas Martínez in Rivas Martínez \& Costa 1976) en la Península Ibérica. Lazaroa 24: 115-116.

BARRERA I. y S. CIRUJANO -1986- ArtemisioPuccinellietum pungentis una nueva asociación de la clase Juncetea maritimi. Trab. Dpt. Bot. Univ. Complut. Madrid 13: 111-113.

CASTROVIEJO S \& J. PORTA -1976- Apport l'ecologie de la vegetation des zones salees des rives de la Cigüela (Ciudad RealEspagne). Colloques Phytosoc. 4: 115-139.

GÉHU, J.M. \& S. RIVAS-MARTÍNEZ -1982Notions fondamentales de Phytosociologie. Ber. Internat. Symp. IVVS, -Syntaxonomie: 1-33. J. Cramer ed.

JULIÀ M.A. -1992-El gènere Puccinellia Parl. a la Península Ibérica. Col.lecció de Tesis Doctorals microfitxedes, núm. 1462.Universitat de Barcelona.

RIVAS MARTÍNEZ, S., F. FERNÁNDEZGONZÁlEZ, J. LOIDI, M. LOUSÃ \& A. PENAS -2001- Syntaxonomical checklist of vascular plant communities of Spain and Portugal to association Level. Itinera Geobotanica 14:5-341.

WEBER, H. E.; J. MORAVEC \&, J. P THÉURILLAT -2000- International Code of Phytosociological Nomenclature. Journal Veg. Sci. 11(5): 739-768.

Aceptado para su publicación en septiembre de 2004

Dirección de los autores: M. A. Alonso \& A. De la Torre. Dpto. Ciencias Ambientales y Recursos Naturales, Universidad de Alicante, Apartado 99, E-03080 Alicante, España. MA.Alonso@ua.es; A.delatorre@ua.es 